\title{
Isolation and Identification of Two Antrodia cinnamomea Strains from Fruiting Bodies
}

\author{
CHIEN-YU CHANG ${ }^{1,2}$, MING-YONG LUE ${ }^{3}$ AND TZU-MING PAN ${ }^{1 *}$ \\ 1. Institute of Microbiology and Biochemistry, National Taiwan University, 1, Sec. 4, Roosevelt Rd., Taipei 106, Taiwan, R.O.C. \\ 2. Taiwan Sugar Research Institute, Tainan, Taiwan \\ 3. Department of Cosmetic Science, Chia-Nan University of Pharmacy and Science, Taiwan
}

(Received: January 26, 2005; Accepted: November 7, 2005)

\begin{abstract}
Antrodia cinnamomea strains ACTS1 and AC0623 were isolated from the fruiting bodies. The partial sequences of ribosomal RNA gene, including the internal transcribed spacers, ITS1-5.8S-ITS2, and 18S ribosomal RNA genes, have been sequenced and applied to identify these two strains. Four specific primer sets (NS1/NS2, NS3/NS4, NS5/NS8, and ITS1/ITS4) were utilized to perform PCR experiment of the ribosomal RNA genes. Comparison of partial nucleotide sequences (ITS1-5.8S-ITS2) with strains from different genus (Antrodiella semisupina, Antrodiella romellii and Trametes versicolor) was made. These DNA sequence data demonstrated that there is no difference among ACTS1, AC0623, BCRC 35396 and BCRC 35398. The colony morphology and growth characteristics of Antrodia cinnamomea strains in different culture media of agar plate were also made. No obvious microscopic difference of A. cinnamomea strains ACTS1, AC0623, BCRC 35396, and BCRC 35398 were observed. The results from this study suggested that strains ACTS1 and AC0623 isolated from fruiting bodies, and the strains BCRC 35396 and 35398 , the original A. cinnamomea strain obtained from Food Industry Research and Development Institute (FIRDI) are the same species.
\end{abstract}

Key words: Antrodia cinnamomea, ribosomal RNA genes, polymerase chain reaction (PCR), fruiting bodies

\section{INTRODUCTION}

Antrodia cinnamomea, a native species in Taiwan, grows naturally on Cinnamomum kanehirai Hay, a kind of camphor tree grown only in Taiwan, and is commonly known as "Niu-chang-chih". This fungus forms fruit bodies that causes brown rot on C. kanehirai ${ }^{(1,2,3)}$. Aborigines living in Taiwan's mountain range have used this fungus for the treatment of food and drug intoxications, diarrhea, abnormal pains, hypertension and liver cancer in Taiwanese folk medicine ${ }^{(2,4,5,6)}$. The current price of fresh fruiting bodies of $A$. cinnamomea is estimated to be US\$ 1500 per $\mathrm{kg}$.

Economic incentives are effective for the development of artificial cultivation methods. Some organizations claim to have induced formation of fruiting bodies since early 2001. However, it seems that growth under such artificial environments still depends on luck. Nevertheless, submerged mycelia products have been recently marketed. We have isolated two strains ACTS1 and AC0623 from fruiting bodies of $A$. cinnamomea at Liou Kuei in southern Taiwan. In order to understand whether strains ACTS1 and AC0623 cultivated in Taiwan Sugar Research Institute (TSRI) and the BCRC 35396 and 35398 strains are the same, some molecular systematic techniques were made ${ }^{(7-22)}$. Random amplified polymorphic DNA (RAPD) markers with polymerase chain reaction (PCR)

\footnotetext{
* Author for correspondence. Tel: +886-2-33664519;

Fax: +886-2-2362-7044; E-mail: tmpan@ntu.edu.tw
}

amplification of genomic DNA technique were also used for identification of Cordyceps species ${ }^{(17,23,24)}$.

Based on PCR fragments of the whole ribosomal RNA gene (including the internal transcribed spacers [ITS1 and ITS2], plus 5.8S, 18S, and 28S ribosomal RNA genes) we can distinguish whether these strains have the same characteristics from the original species. The results of isolation and identification of these strains are described as follows.

\section{MATERIALS AND METHODS}

\section{Strains of Antrodia cinnamomea}

The strains of this study $A$. cinnamomea BCRC 35396 and A. cinnamomea BCRC 35398 were purchased from Bioresources Collection \& Research Center (BCRC, FIRDI, Hsinchu, Taiwan). Strains of ACTS1 and AC0623 were isolated from fruiting bodies of $A$. cinnamomea at Liou Kuei in southern Taiwan as illustrated in Table 1.

\section{Isolation of Two Antrodia cinnamomea Strains from Fruiting Bodies}

Firstly the fruiting body was treated with antibiotics penicillin $\mathrm{V}$ or ampicillin to inhibit the growth of contaminants. Then it was cut into small pieces, put into the sterilized water and isolated from different dilutions of sterilized water on agar plate. Colonies were grown 
on plate for three weeks on M25 plate. Two strains of ACTS1 and AC0623 were isolated from fruiting bodies of $A$. cinnamomea.

\section{Antrodia cinnamomea Cultured Media}

A. cinnamomea strains ACTS1, AC0623, BCRC 35396, and BCRC 35398 were cultured and maintained at $28^{\circ} \mathrm{C}$ on M25 agar plate (Merck, Germany). Mycelium obtained from the edge of 21-day-old colonies was used as the inoculum source. Other culture media such as PDA (potato dextrose agar, Difco, Becton Dickinson, USA), OAT (oatmeal agar, Difco, Becton Dickinson, USA), SGA (Sabouraud-2\% glucose agar, Merck, Germany), and NA (nutrient agar, Merck, Germany) agar plate were used to make a comparison of colony growth. Cell mass production was carried out in 500-mL Hinton flask with rotary shaker of $110 \mathrm{rpm}$ and $28^{\circ} \mathrm{C}$ respectively. The M25 cultured media of $A$. cinnamomea strains are as follows: glucose $3 \%$, bacto-peptone $1.5 \%$, yeast extract $1.5 \%$, malt extract $1.5 \%, \mathrm{MgSO}_{4} \cdot 7 \mathrm{H}_{2} \mathrm{O} 0.03 \%, \mathrm{KH}_{2} \mathrm{PO}_{4} 0.03 \%$ and $\mathrm{K}_{2} \mathrm{HPO}_{4} 0.03 \%$, pH 5.5 .

\section{Extraction of Genomic DNA}

Genomic DNA was prepared using plant DNAzol Reagent $^{(25)}$ (Invitrogen, USA). The procedures were as follows.

\section{(I) Extration}

Plant tissues of $A$. cinnamomea were pulverized in liquid nitrogen using mortar and pestle. As the samples were grinded, liquid nitrogen was replenished in the mortar 2 to 3 times. The resulting homogenous frozen powder was then transferred to a microcentrifuge tube containing plant DNAzol. Every gram of plant tissue needed $3 \mathrm{~mL}$ of plant DNAzol. The mixture was mixed thoroughly by gentle inversion a few times before incubation at $25^{\circ} \mathrm{C}$ and shaking for 5 min. The mixture was then added to $0.3 \mathrm{~mL}$ of chloroform and mixed vigorously. After incubation at $25^{\circ} \mathrm{C}$ for another $5 \mathrm{~min}$, the mixture was centrifuged.

\section{(II) Centrifugation}

Following extraction, the extract was centrifuged at $12,000 \times \mathrm{g}$ for $10 \mathrm{~min}$. The supernatant was transfer to a fresh tube.

\section{(III) DNA Precipitation}

The collected supernatant was mixed with 0.23 $\mathrm{mL}$ of absolute ethanol. The compound was mixed by inverting the tube 6 to 8 times before storage at room temperature for $5 \mathrm{~min}$. Precipitated DNA was separated by centrifugation at $5,000 \times \mathrm{g}$ for $4 \mathrm{~min}$.
Table 1. Source and collection number of Antrodia cinnamomea strains in this study

\begin{tabular}{ccc}
\hline Species & Collection number & Source \\
\hline Antrodia cinnamomea & BCRC 35396 & BCRC $^{\mathrm{a}}$, Taiwan \\
Antrodia cinnamomea & BCRC 35398 & BCRC, Taiwan \\
Antrodia cinnamomea & ACTS1 & $\begin{array}{c}\text { Liou Kuei, Taiwan } \\
\text { isolated by TSRI } \\
\text { fruiting body } \\
\text { Taiwan }\end{array}$ \\
$\begin{array}{c}\text { Antrodia cinnamomea } \\
\text { fruiting body }\end{array}$ & AC0623 & $\begin{array}{c}\text { Liou Kuei, Taiwan, } \\
\text { isolated by TSRI, } \\
\text { Taiwan }\end{array}$ \\
\hline
\end{tabular}

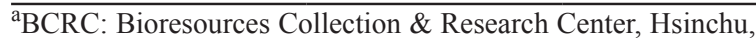
Taiwan.

${ }^{\mathrm{b}}$ TSRI: Taiwan Sugar Research Institute, Tainan, Taiwan.

Table 2. Primers used in this study ${ }^{(21)}$

\begin{tabular}{ccc}
\hline $\begin{array}{c}\text { Primer } \\
\text { designation }\end{array}$ & Primer sequences $\left(5^{\prime} \rightarrow 3^{\prime}\right)$ & Tm $\left({ }^{\circ} \mathrm{C}\right)$ \\
\hline NS1 & GTAGTCATATGGCTTGTCTC & 54 \\
NS2 & GGCTGCTGGCACCAGACTTGC & 62 \\
NS3 & GCAAGTCTGGTGCCAGCAGCC & 62 \\
NS4 & CTTCCGTCAATTCCTTTAAG & 56 \\
NS5 & AACTTAAAGGAATTGACGGAAG & 49 \\
NS8 & TCCGCAGGTTCACCTACGGA & 64 \\
ITS1 & TCCGTAGGTGAACCTGCGG & 62 \\
ITS4 & TCCTCCGCTTATTGATATGC & 58 \\
\hline
\end{tabular}

(IV) DNA Wash

\section{Plant DNAzol-ethanol Wash}

Plant DNAzol-ethanol wash was prepared by mixing 1 volume of DNAzol with 0.75 volume of $100 \%$ ethanol. A quantity of $0.3 \mathrm{~mL}$ of Plant DNAzol-ethanol wash solution was mixed with the DNA precipitate by vortexing. After $5 \mathrm{~min}$, the mixture was subjected to centrifugation at 5,000 $\times \mathrm{g}$ for $4 \mathrm{~min}$.

\section{Ethanol Wash}

After the DNAzol wash solution was removed, the DNA pellet was washed by vigorously mixing with 0.3 $\mathrm{mL}$ of $75 \%$ ethanol. The mixture was then centrifuged at $5,000 \times \mathrm{g}$ for $4 \mathrm{~min}$.

\section{(V) DNA Solubilization}

After ethanol layer was removed by decanting, the remaining ethanol was further removed with a micropipette that was allowed to sit vertically for about 2 min. The remaining DNA pellet was air dried and then dissolved in $70 \mu \mathrm{L}$ of TE buffer $(10 \mathrm{mM}$ Tris- $\mathrm{HCl}, 1 \mathrm{mM}$ EDTA, pH 8.0). If DNA pellet is difficult to dissolve, 8 $\mathrm{mM} \mathrm{NaOH}$ solution can be used instead of TE buffer. In a typical DNA preparation, the DNA solution is cloudy and 
may contain insoluble material, which can be removed by centrifugation at $12,000 \times \mathrm{g}$ for $4 \mathrm{~min}$.

\section{Polymerase Chain Reaction (PCR) of rRNA Gene}

The fragment of ITS1-5.8S-ITS2, 18S rRNA
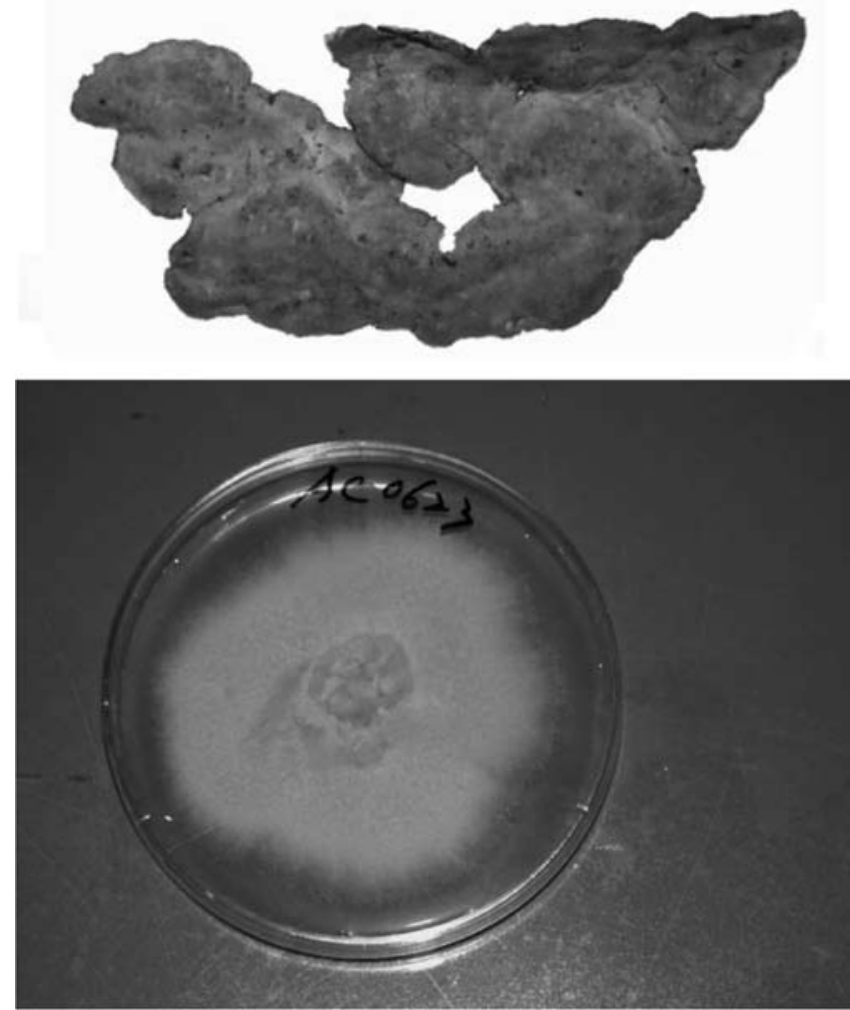

Figure 1. The fruiting body of Antrodia cinnamomea strain AC0623 and colonies grow 3 weeks on M25 agar plate. gene were amplified by polymerase chain reaction. The quantitative analysis of DNA was obtained using OD260/280 after extraction of genomic DNA. Fifty ng of DNA samples from each strain were taken, and the concentration of $\mathrm{MgCl}_{2}$ is $1.5 \mathrm{mM}$. Four specific primer sets (NS1\&NS2, NS3\&NS4, NS5\&NS8, and ITS1\&ITS4) were utilized to perform PCR experiments of the ribosomal RNA genes. Table 2 shows the primers used in this study. The reaction condition of PCR is shown as follows: reaction volume: $100 \mu \mathrm{L}$, dNTP concentration: $200 \mu \mathrm{M}$, primer concentration: $0.5 \mu \mathrm{M}$, and dosage of Taq polymerase (Promega, USA): $0.1 \mathrm{U} /$ $\mu \mathrm{L}$. The procedure of PCR reaction was began with $95^{\circ}$ $\mathrm{C}$ for $5 \mathrm{~min}$. and then heated for $1 \mathrm{~min}$. for denaturation. The annealing temperature is $57^{\circ} \mathrm{C}$ (for $1 \mathrm{~min}$ ) and the elongation temperature is $72^{\circ} \mathrm{C}$ (for $2 \mathrm{~min}$ ) for 40 cycles. The PCR products were separated by electrophoresis in a $2.0 \%$ agarose gel, $100 \mathrm{~V}$ voltage in 0.5 times TBE (50 $\mathrm{mM}$ Tris base, $45 \mathrm{mM}$ boric acid, and $0.5 \mathrm{mM}$ EDTA) buffer (Invitrogen, USA) and then stained with ethidium bromide. The gel was observed and photographed under ultraviolet light.

\section{RESULTS}

I. Isolation of Two Antrodia cinnamomea Strains from Fruiting Bodies

Figure 1 shows the fruiting body of $A$. cinnamomea strain AC0623 and colonies grew 3 weeks on M25 agar plate (Merck, Germany). The comparison of strains ACTS1, AC0623, BCRC 35396 and BCRC 35398 on M25, PDA, OAT, SGA, and NA agar plate are shown in Table 3.
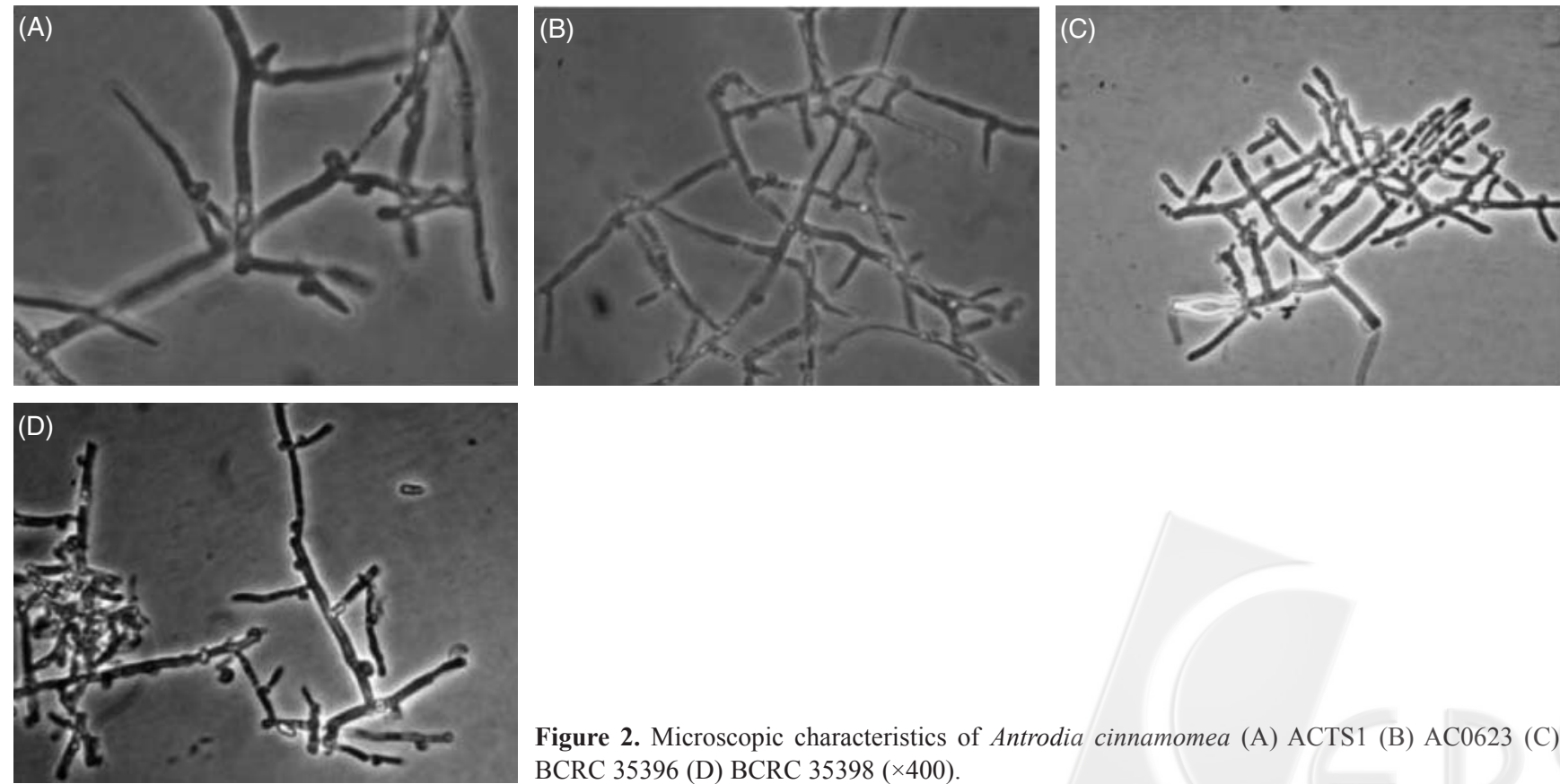

Figure 2. Microscopic characteristics of Antrodia cinnamomea (A) ACTS1 (B) AC0623 (C) BCRC 35396 (D) BCRC $35398(\times 400)$. 

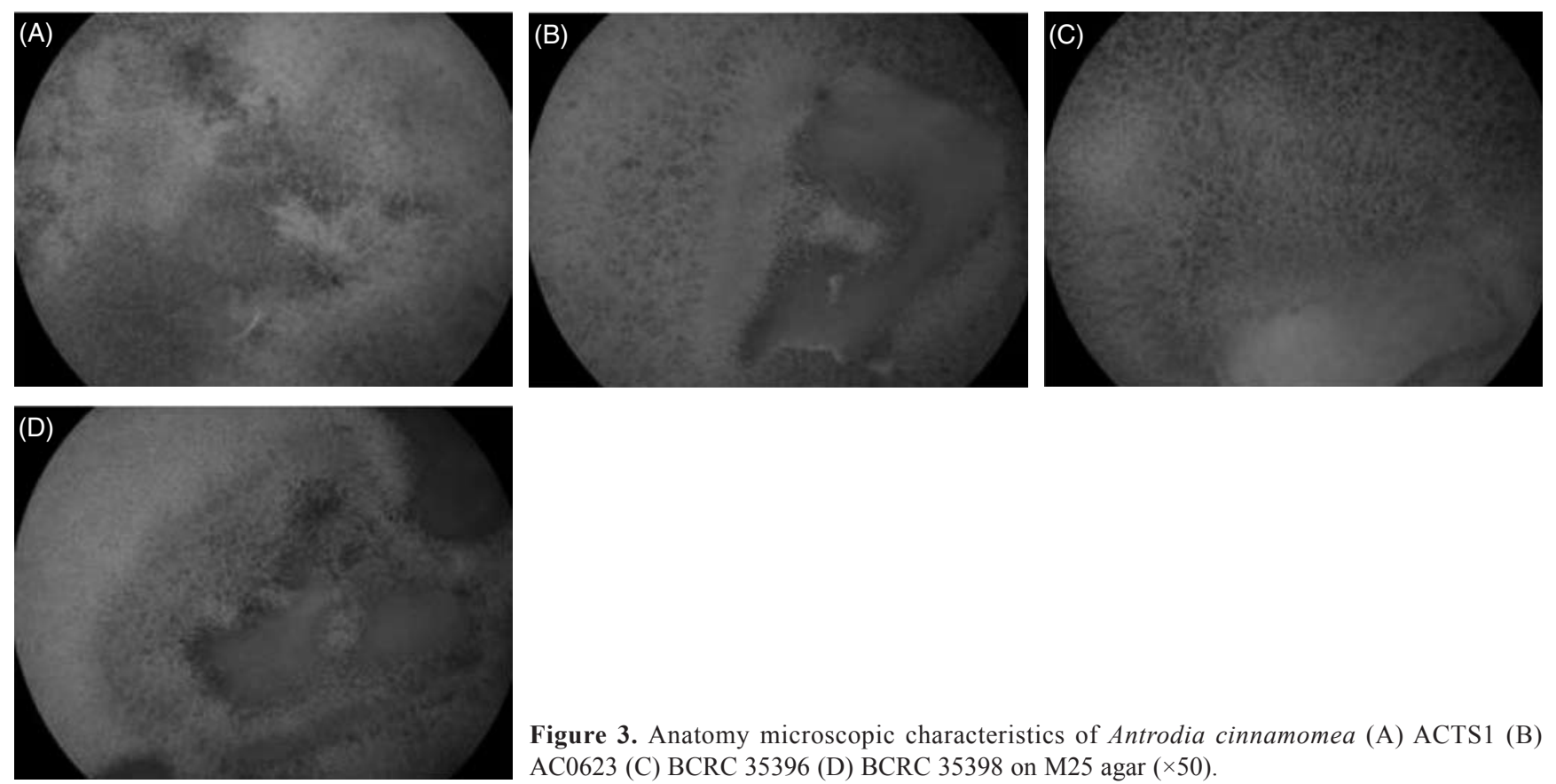

Figure 3. Anatomy microscopic characteristics of Antrodia cinnamomea (A) ACTS1 (B) AC0623 (C) BCRC 35396 (D) BCRC 35398 on M25 agar (×50).

Table 3. Diameter of the colonies of Antrodia cinnamomea strains in different media

\begin{tabular}{|c|c|c|c|c|}
\hline Medium & ACTS1 & AC0623 & $\begin{array}{l}\text { BCRC } \\
35396\end{array}$ & $\begin{array}{l}\text { BCRC } \\
35398\end{array}$ \\
\hline M25 & $5.0 \mathrm{~cm}^{\mathrm{a}}$ & $6.5 \mathrm{~cm}$ & $6.0 \mathrm{~cm}$ & $5.0 \mathrm{~cm}$ \\
\hline PDA & $4.5 \mathrm{~cm}$ & $5.5 \mathrm{~cm}$ & $5.5 \mathrm{~cm}$ & $5.3 \mathrm{~cm}$ \\
\hline OAT & $4.5 \mathrm{~cm}$ & $4.5 \mathrm{~cm}$ & $5.3 \mathrm{~cm}$ & $6.5 \mathrm{~cm}$ \\
\hline SGA & $4.3 \mathrm{~cm}$ & $4.5 \mathrm{~cm}$ & $4.0 \mathrm{~cm}$ & $5.0 \mathrm{~cm}$ \\
\hline NA & $-^{\mathrm{b}}$ & - & - & - \\
\hline
\end{tabular}

${ }^{\mathrm{a}} \mathrm{cm}$ : the length were measured when the colonies reached their maximum diameter of A. cinnamomea strains within 21 days after inoculated on the plates.

${ }^{\mathrm{b}}$ No growth.

The growth rate of $A$. cinnamomea on M25 agar was the fastest among all combination tested. The microscopic traits of these strains are shown in Figure 2, and the anatomy microscopic characteristics of $A$. cinnamomea are depicted in Figure 3.

II. Comparison of the PCR Products of Ribosomal RNA Genes among the Antrodia cinnamomea Strain ACTS1, $A C 0623, B C R C 35396$, and BCRC 35398

Four specific primer sets (NS1/NS2, NS3/NS4, NS5/NS8, and ITS1/ITS4) were utilized to perform PCR amplification of the ribosomal RNA genes (including 18S and ITS1-5.8S-ITS2 rRNA gene). The results are shown in Figure 4. The fragment length and location with specific primer sets to perform PCR amplification are shown as follows:

The PCR product of NS1 \& NS2: 550 bp (18S rRNA gene) The PCR product of NS3 \& NS4: $600 \mathrm{bp}$ (18S rRNA gene) The PCR product of NS5 \& NS8: 650 bp (18S rRNA gene)

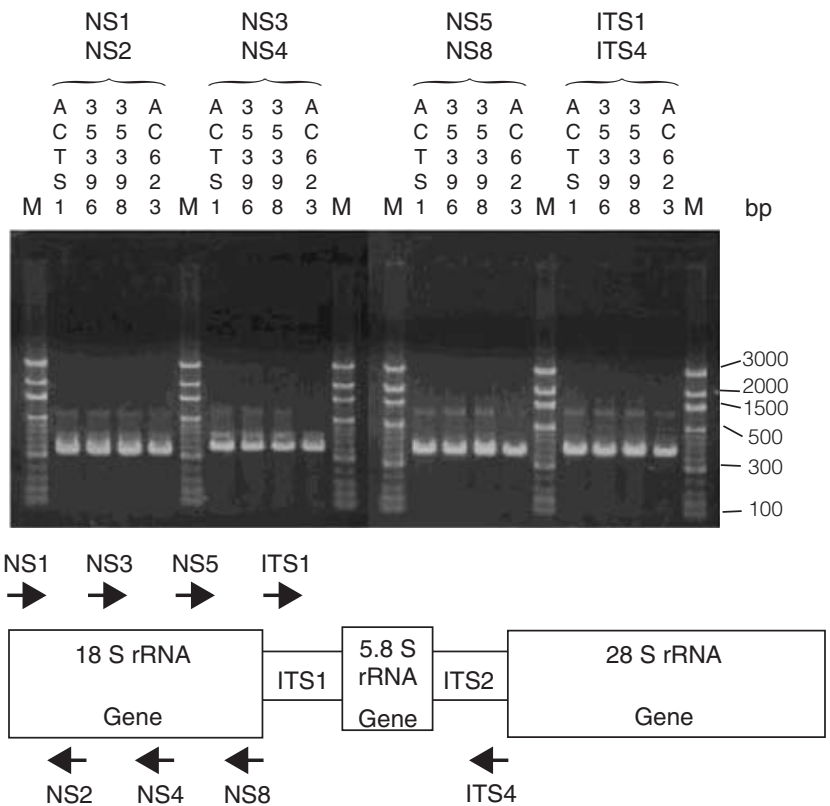

Figure 4. Comparison of the PCR products of ribosomal RNA genes among the Antrodia cinnamomea strain ACTS1, AC0623 (TSRI), and BCRC 35396, BCRC 35398 strain (FIRDI). PCR products were separated by electrophoresis in a $2.0 \%$ agarose gel and then stained with ethidium bromide. The gel was observed and photographed under ultraviolet light. The100-bp ladder (M) as molecular weight standards is shown in intervals.

The PCR product of ITS1\& ITS4: 700 bp (ITS1-5.8S-ITS2 rRNA gene)

III. DNA Sequence Analysis of Antrodia cinnamomea Strain $^{(16)}$ and Others

Four specific primer sets (NS1/NS2, NS3/NS4, NS5/ 
NS8, and ITS1/ITS4) were utilized to perform primer for direct DNA sequence of PCR product. Ribosomal RNA genes of ACTS1, BCRC 35396, BCRC 35398, and

Table 4. Similarity of DNA sequences of partial ribosomal RNA genes [containing partial $18 \mathrm{~S}$ ribosomal RNA gene, internal transcribed spacer 1 (ITS1), 5.8S ribosomal RNA gene, internal transcribed spacer 2 (ITS2), and partial $28 \mathrm{~S}$ ribosomal RNA gene] among 4 Antrodia cinnamomea strains including ACTS1 (AY378092), AC0623 (AY378093), BCRC 35396 (AY378094), and BCRC 35398 (AY378095)

\begin{tabular}{ccccc}
\hline Strain & AC0623 & $\begin{array}{l}\text { BCRC } \\
35396\end{array}$ & $\begin{array}{l}\text { BCRC } \\
35398\end{array}$ & ACTS1 \\
\hline ACTS1 & $100 \%$ & $99.8 \%$ & $99.9 \%$ & $100 \%$ \\
AC0623 & $100 \%$ & $99.8 \%$ & $99.9 \%$ & $100 \%$ \\
BCRC & $99.8 \%$ & $100 \%$ & $99.9 \%$ & $99.8 \%$ \\
35396 & & & & \\
BCRC & $99.9 \%$ & $99.9 \%$ & $100 \%$ & $99.9 \%$ \\
35398 & & & & \\
\hline
\end{tabular}

AC0623 were used for DNA sequence analysis. Based on the ribosomal RNA genes (18S and ITS1-5.8S-ITS2 rRNA genes) and four specific primer sets, the length of PCR product after reaction was $556 \mathrm{bp}, 602 \mathrm{bp}, 659 \mathrm{bp}$ and $682 \mathrm{bp}$ respectively. The complete sequence of $18 \mathrm{~S}$ rRNA, ITS1, 5.8S rRNA and ITS2 were obtained. The results of the DNA sequences analysis among ACTS1, AC0623, BCRC 35396, and BCRC 35398 strains are shown in Table 4.

From the sequence analysis of Antrodia cinnamomea $^{(26)}$, $18 \mathrm{~S}$ rRNA is shown to be a high conservative region (about $1700 \mathrm{bp}$ ) and the similarity between BCRC 35396 and BCRC 35398 strains was over $98 \%$. Since the region of partial nucleotide sequence ITS1-5.8S-ITS2 (about $680 \mathrm{bp}$ ) is non-conservative evolutionally, it is suitable for species identification. Partial nucleotide sequences (ITS1-5.8S-ITS2) were compared with those of the strains from different genus (Antrodiella semisupina, Antrodiella romellii, and Trametes versicolor). The sequences were analyzed
BCRC35396

BCRC35716

AC0623

ACTS1

BCRC35398

Antrodiella semisupina

Antrodiella romellii

Trametes versicolor

BCRC35396

BCRC35716

AC0623

ACTS1

BCRC35398

Antrodiella semisupina

Antrodiella romellii

Trametes versicolor

BCRC35396

BCRC35716

AC0623

ACTS1

BCRC35398

Antrodiella semisupina

Antrodiella romellii

Trametes versicolor

BCRC35396

BCRC 35716

AC0623

ACTS1

BCRC35398

Antrodiella semisupina

Antrodiella romellii

Trametes versicolor
TCCGTAGGTGAACCTGCGGAAGGACCATTATTGTAT----TTGAAAGGGG TCCGTAGGTGAACCTGCGGAAGGACCATTATTGTAT-----TTGAAAGGGG TCCGTAGGTGAACCTGCGGAAGGATCATTATTGTAT----TTGAAAGGGG TCCGTAGGTGAACCTGCGGAAGGATCATTATTGTAT----TTGAAAGGGG TCCGTAGGTGAACCTGCGGAAGGATCATTATTGTAT----TTGAAAGGGG TCCGTAGGTGAACCTGCGGAAGGATCATTAATGAATGAACTTGGGCATAG TCCGTAGGTGAACCTGCGGAAGGATCATTAATGAATGAACTTGGGCAAAG TCCGTAGGTGAACCTGCGGAAGGATCATTAACGAGT---TTTGAAACGAG

TTGTAGCTGACCTCCTCTTGAAAAGGGGGGAGGTATGTGCACACCTCT-G TTGTAGCTGACCTCCTCTTGAAAAGGGGGGAGGTATGTGCACACCTCTTG TTGTAGCTGACCTCCTCTTGAAAAGGGGGGAGGTATGTGCACACCTCT-G TTGTAGCTGACCTCCTCTTGAAAAGGGGGGAGGTATGTGCACACCTCT-G TTGTAGCTGACCTCCTCTTGAAAAGGGGGGAGGTATGTGCACACCTCT-G TTGTAGCTGGCCTC--------AGCAATGAGGCATGTGCACAC-TGT-G TTGTAGCTGGCCTC---------AGCAATGGGGCATGTGCACAC-TTT-G TTGTAGCTGGCCTT---------C----CGGGGCATGTGCACGC-TCT-G

TTCATTCATATTCTCTCACACCTGTGCATGCTTTGTAGGTTGGTTTTGAA TTCATTCATATTCTCTCACACCTGTGCATGCTTTGTAGGTTGGTTTTGAA TTCATTCATATTCTCTCACACCTGTGCATGCTTTGTAGGTTGGTTTTGAA TTCATTCATATTCTCTCACACCTGTGCATGCTTTGTAGGTTGGTTTTGAA TTCATTCATATTCTCTCACACCTGTGCATGCTTTGTAGGTTGGTTTTGAA TTCATCCAC-CCTTCACACCTCTGTGCACTTCTCATGGGTTTGGGTCAAG TTCATCCAC-C-TTCACACCACTGTGCACTTCTCATGGGTC-GGGTTGCG CTCATCCAC-TCT----ACCCCTGTGCACTTACTGTAGGTTGGCGTGGGC

TGGTTGTCTTCTCTGATGGAGACAGCTGTTTTGACCTTCCTATGTTTTTT TGGTTGTCTTCTCTGATGGAGACAGCTGTTTTGACCTTCCTATGTTTTTT TGGTTGTCTTCTCTGATGGAGACAGCTGTTTTGACCTTCCTATGTTTTTT TGGTTGTCTTCTCTGATGGAGACAGCTGTTTTGACCTTCCTATGTTTTTT TGGTTGTCTTCTCTGATGGAGACAGCTGTTTTGACCTTCCTATGTTTTTT T--------CTGAAATATGGCGAAGCCC-----CCTTCTCATGTGTTTT

T--------CTGAAATATGGCAAAGCCC-------TTCTCATGTGTTTT

T--------CCTTAACGGGAGCATTCTG-----CCGGCCTATGTATACT 
BCRC35396

BCRC35716

AC0623

ACTS1

BCRC35398

Antrodiella semisupina

Antrodiella romellii

Trametes versicolor

BCRC35396

BCRC35716

AC0623

ACTS1

BCRC35398

Antrodiella semisupina

Antrodiella romellii

Trametes versicolor

BCRC35396

BCRC35716

AC0623

ACTS1

BCRC35398

Antrodiella semisupina

Antrodiella romellii

Trametes versicolor

BCRC35396

BCRC35716

AC0623

ACTS1

BCRC35398

Antrodiella semisupina

Antrodiella romellii

Trametes versicolor

BCRC35396

BCRC35716

AC0623

ACTS1

BCRC35398

Antrodiella semisupina

Antrodiella romellii

Trametes versicolor

BCRC35396

BCRC35716

AC0623

ACTS1

BCRC35398

Antrodiella semisupina

Antrodiella romellii

Trametes versicolor
AAATTGACTCCGTATCAGTTACAGAATGTA----TGTTGCGTGTAACGCA AAATTGACTCCGTATCAGTTACAGAATGTA----TGTTGCGTGTAACGCA AAATTGACTCCGTATCAGTTACAGAATGTA----TGTTGCGTGTAACGCA AAATTGACTCCGTATCAGTTACAGAATGTA----TGTTGCGTGTAACGCA AAATTGACTCCGTATCAGTTACAGAATGTA----TGTTGCGTGTAACGCA AAACACACACTATACAAGTTTTAGAATGTAACAATCATGCGTC-AATGCA AC-CACACACT--ACAAGTTTTAGAATGTAACAATCATGCATT-AATGCA ACAAACACTTTA---AAGTATCAGAATGTAA-----ACGCGTCTAACGCA

T--ATTGTATAACTTTCAGCAACGGATCTCTTGGCTCTCGCATCGATGAA T--ATTGTATAACTTTCAGCAACGGATCTCTTGGCTCTCGCATCGATGAA T--ATTGTATAACTTTCAGCAACGGATCTCTTGGCTCTCGCATCGATGAA T--ATTGTATAACTTTCAGCAACGGATCTCTTGGCTCTCGCATCGATGAA T--ATTGTATAACTTTCAGCAACGGATCTCTTGGCTCTCGCATCGATGAA T--ATAATACAACTTTCAGCAACGGATCTCTTGGCTCTCGCATCGATGAA T--ATAATACAACTTTCAGCAACGGATCTCTTGGCTCTCGCATCGATGAA TCTATAATACAACTTTTAGCAACGGATCTCTTGGCTCTCGCATCGATGAA

GAACACGGCGAAATGTGATAAGTAATGTGAATTGCAGAATTCAGTGAATC GAACACGGCGAAATGTGATAAGTAATGTGAATTGCAGAATTCAGTGAATC GAACACGGCGAAATGTGATAAGTAATGTGAATTGCAGAATTCAGTGAATC GAACACGGCGAAATGTGATAAGTAATGTGAATTGCAGAATTCAGTGAATC GAACACGGCGAAATGTGATAAGTAATGTGAATTGCAGAATTCAGTGAATC GAACGCAGCGAAATGCGATAAGTAATGTGAATTGCAGAATTCAGTGAATC GAACGCAGCGAAATGCGATAAGTAATGTGAATTGCAGAATTCAGTGAATC GAACGCAGCGAAATGCGATAAGTAATGTGAATTGCAGAATTCAGTGAATC

ATCGAATCTTTGAACGCACCTTGCGCTCCTTGGTATTCTGAGGAGCATGC ATCGAATCTTTGAACGCACCTTGCGCTCCTTGGTATTCTGAGGAGCATGC ATTGAATCTTTGAACGCACCTTGCGCTCCTTGGTATTCTGAGGAGCATGC ATTGAATCTTTGAACGCACCTTGCGCTCCTTGGTATTCTGAGGAGCATGC ATCGAATCTTTGAACGCACCTTGCGCTCCTTGGTATTCTGAGGAGCATGC ATCGAATCTTTGAACGCACCTTGCGCTCCTTGGTATTCCGAGGAGCATGC ATCGAATCTTTGAACGCACCTTGCGCTCCTTGGTATTCCGAGGAGCATGC ATCGAATCTTTGAACGCACCTTGCGCTCCTTGGTATTCCGAGGAGCATGC

CTGTTTGAGTGTCATGGAATTATCAACCCTTTTGACTTTTTGT------CTGTTTGAGTGTCATGGAATTATCAACCCTTTTGACTTTTTGT-----CTGTTTGAGTGTCATGGAATTATCAACCCTTTTGACTTTTTGT------CTGTTTGAGTGTCATGGAATTATCAACCCTTTTGACTTTTTGT------CTGTTTGAGTGTCATGGAATTATCAACCCTTTTGACTTTTTGT------CTGTTTGAGTGTCATGGTATTCTCAACCCTGCTACATTTTTTTGAAAGAT CTGTTTGAGTGTCATGGTATTCTCAACCCTGCTACATTTTTT-------CTGTTTGAGTGTCATGGAATTCTCAACTT--ATAAATCCTTGTG------

-TGAATGGGCTTGGATTTGGAGGGTTAAATTGCTGGCTCTT----CTTTT -TGAATGGGCTTGGATTTGGAGGGTTAAATTGCTGGCTCTT----TTTTT -TGAATGGGCTTGGATTTGGAGGGTTAAATTGCTGGCTCTT----TTTTT -TGAATGGGCTTGGATTTGGAGGGTTAAATTGCTGGCTCTT----TTTTT -TGAATGGGCTTGGATTTGGAGGGTTAAATTGCTGGCTCTT----TTTTT GTTGCTTGGCTTGGACTTGGAGGTTT--ATTGCTGGCATTCAACTTGTTT GTAGCTGGGCTTGGACTTGGAGGT----ATTGCCGGTGTTC---TCTTTT ATCTATAAGCTTGGACTTGGAGGC-----TTGCTGGCCCTT-- 
BCRC35396

BCRC35716

AC0623

ACTS1

BCRC35398

Antrodiella semisupina

Antrodiella romellii

Trametes versicolor

BCRC35396

BCRC35716

AC0623

ACTS1

BCRC35398

Antrodiella semisupina

Antrodiella romellii

Trametes versicolor

BCRC35396

BCRC35716

AC0623

ACTS1

BCRC35398

Antrodiella semisupina

Antrodiella romellii

Trametes versicolor

BCRC35396

BCRC35716

AC0623

ACTS1

BCRC35398

Antrodiella semisupina

Antrodiella romellii

Trametes versicolor

$\begin{array}{ll}\text { BCRC35396 } & \text { GAGGA } \\ \text { BCRC35716 } & \text { GAGG- } \\ \text { AC0623 } & \text { GAGGA } \\ \text { ACTS1 } & \text { GAGGA } \\ \text { BCRC35398 } & \text { GAGGA } \\ \text { Antrodiella semisupina } & \text { GAGGA } \\ \text { Antrodiella romellii } & \text { GAGGA } \\ \text { Trametes versicolor } & \text { GAGGA }\end{array}$

GAGGA
GAGG-
GAGGA
GAGGA
GAGGA
GAGGA
GAGGA
GAGGA

T-GATTCAGCTCCTCTTGAATGCATTAGCTTGAACCCTTGTGGATTGACC T-GATTCAGCTCCTCTTGAATGCATTAGCTTGAACCCTTGTGGATTGACC TTGATTCAGCTCCTCTTGAATGCATTAGCTTGAACCCTTGTGGATTGACC TTGATTCAGCTCCTCTTGAATGCATTAGCTTGAACCCTTGTGGATTGACC T-GATTCAGCTCCTCTTGAATGCATTAGCTTGAACCCTTGTGGATTGACC GAACGCGGGCTCCTCTGAAATGCATTAGCTGGAATGT-TACCGAGCATGA GAACGCGGGCTCCTCTGAAATGCATTAGCTGGAATGT-TACCGAGCATGA -GCGGTCGGCTCCTCTTGAATGCATTAGCTTGATTCCGTACGGATCGGCT

TTATCGGTGTGATAGTCATCTATGCCGTGGTTGTCTGAGGTGGGATCGGC TTATCGGTGTGATAGTCATCTATGCCGTGGTTGTCTGAGGTGGGATCGGC TTATCGGTGTGATAGTCATCTATGCCGTGGTTGTCTGAGGTGGGATCGGC TTATCGGTGTGATAGTCATCTATGCCGTGGTTGTCTGAGGTGGGATCGGC TTATCGGTGTGATAGTCATCTATGCCGTGGTTGTCTGAGGTGGGATCGGC TT--CAATGTGATAATTGTCTACGTTGCTTCAACTTGGTATTAATTCTGT TT--CAATGTGATAATTGTCTACGTTGCTTCAACTTGGTATTAATTCTGT CT--CAGTGTGATAATTGTCTACGCTGT---GACCGTGAAGTGTTTTGGC

TTCTAATGGTGCAAGTCCCTTCAGGGGGATGATTTTCTAATGACCTTCTG TTCTAATGGTGCAAGTCCCTTCAGGGGGATGATTTTCTAATGACCTTCTG TTCTAATGGTGCAAGTCCCTTCAGGGGGATGATTTTCTAATGACCTTCTG TTCTAATGGTGCAAGTCCCTTCAGGGGGATGATTTTCTAATGACCTTCTG TTCTAATGGTGCAAGTCCCTTCAGGGGGATGATTTTCTAATGACCTTCTG TTCAGCTTCTAACCGTCCCCTTTGCGGGACAATATCT---TGAACATCTG TTCAGCTTCTAACCGTCCGCA----AGGACAATATCT---TGAACATCTG --GAGCTTCTAACCGTCCATT----AGGACAACTTCT---T-AACATCTG

ACCTCAAATCAGGTAGGACTACCCGCTGAACTTAAGCATATCAATAAGCG ACCTCAAATCAGGTAGGACTACCCGCTGAACTTAAGCATATCAATAAGCG ACCTCAAATCAGGTAGGACTACCCGCTGAACTTAAGCATATCAATAAGCG ACCTCAAATCAGGTAGGACTACCCGCTGAACTTAAGCATATCAATAAGCG ACCTCAAATCAGGTAGGACTACCCGCTGAACTTAAGCATATCAATAAGCG ACCTCAAATCAGGTAGGACTACCCGCTGAACTTAAGCATATCAATAAGCG ACCTCAAATCAGGTAGGACTACCCGCTGAACTTAAGCATATCAATAAGCG ACCTCAAATCAGGTAGGACTACCCGCTGAACTTAAGCATATCAATAAGCG

Figure 5. Comparison of DNA sequences of partial ribosomal RNA genes (containing partial ITS1, 5.8S rRNA gene, and partial ITS2) among 5 Antrodia cinnamomea strains including ACTS1 (AY378092), AC0623 (AY378093), BCRC 35396 (AY378094), BCRC 35398 (AY378095), and BCRC 35716 (AJ496402), as well as Antrodiella semisupina (AF126903), Antrodiella romellii (AF126902), and Trametes versicolor (AY309015) strains.

and are shown in Figure 5. The results from this study suggest that the two strains (ACTS1 and AC0623) isolated from fruiting bodies are of the same species, $A$. cinnamomea. The nucleotide sequences reported in this and other paper have been submitted to the GenBank with accession numbers AY378092 (ACTS1), AY378093
(AC0623), AY378094 (BCRC 35396), AY378095 (BCRC 35398), AJ496402 (BCRC 35716) ${ }^{(26)}$, AY309015 (Trametes versicolor), AF126902 (Antrodiella romellii) ${ }^{(27)}$, and AF126903 (Antrodiella semisupina) ${ }^{(27)}$. 


\section{DISCUSSION}

PCR primers that target conserved regions of fungal rRNA genes, amplifying sequence-variable fragments of genes or intervening noncoding regions, have been used for sequence comparisons. Interspecies variability is also manifested in the fragment size of the internal transcribed spacer 1 and 2 (ITS1 and ITS2, respectively) regions ${ }^{(28)}$. In order to identify A. cinnamomea rapidly and correctly, the molecular biology methods for A. cinnamomea species and strains analysis were used ${ }^{(26)}$. Firstly, they used 18S rRNA gene, ITS gene and intergenic spacer of rRNA, and Mn-superoxide dismutase gene ${ }^{(26)}$. In addition, phylogenetic analysis methods, Neighbor Joing method, Parsimony method and Maximum Likelihood method were also used to analyze evolution relation of A. cinnamomea $^{(26)}$. The specific primer sets based PCR fragments of the whole ribosomal RNA gene [including the internal transcribed spacers (ITS1 and ITS2), 5.8S, and $18 \mathrm{~S}$ ribosomal RNA genes] combined partial nucleotide sequence (ITS1-5.8S-ITS2) analysis with other genus for negative control could be applied to investigate whether the strain cultivated in Taiwan Sugar Research Institute (TSRI) is the species named A. cinnamomea. Comparison of colony morphology and growth characteristics of Antrodia cinnamomea strains in different culture media of agar plates was made. The growth rate of $A$. cinnamomea on M25 agar was the fastest among all combinations tested. Nutrient agar medium was not suitable for $A$. cinnamomea strains. The results demonstrate that there is no difference in the eletrophoresisgram of the amplified PCR products between strains ACTS1, AC0623, BCRC 35396, and BCRC 35398. No obvious microscopic difference of A. cinnamomea strains ACTS1, AC0623, BCRC 35396, and BCRC 35398 was observed in this study. The major biologically active component triterpenoids will be in conjunction with our continuing research from the cultured A. cinnamomea mycelia.

\section{ACKNOWLEDGEMENTS}

We would like to thank Mr. Shun-Yuan Yang for assisting the PCR experiment in this study.

\section{REFERENCES}

1. Andersson, B. E., Tornberg, K., Henrysson, T. and Olsson, S. 2001. Three-dimensional outgrowth of a wood-rotting fungus added to a contaminated soil from a former gasworks site. Bioresour. Technol. 78: 37-45.

2. Chang, T. T. and Chou, W. N. 1995. Antrodia cinnamomea sp. nov. on Cinnamomea kanehirai in Taiwan. Mycol. Res. 99: 756-758.

3. Wu, S. H., Ryvarden, L. and Chang, T. T. 1997. Antrodia cinnamomea ("niu-chang-chih"), new com- bination of a medicinal fungus in Taiwan. Bot. Bull. Acad. Sin. 38: 273-275.

4. Cherng, I. H. and Chiang, H. C. 1995. Three new triterpenoids from Antrodia cinnamomea. J. Natural. Products 58: 365-371.

5. Hseu, Y. C., Chang, W. C., Hseu, Y. T., Lee, C. Y., Tech, Y. J., Chen, P. C., Chen, J. Y. and Yang, H. L. 2002. Protection of oxidative damage by aqueous extract from Antrodia cinnamomea mycelia in normal human erythrocytes. Life Sci. 71: 469-482.

6. Song, T. Y. and Yen, G. C. 2002. Antioxidant properties of Antrodia cinnamomea in submerged culture. J. Agric. Food Chem. 50: 3322-3327.

7. Buzina, W., Doris, L. L., Braun, H., Freudenschuss, K. and Stammberger, H. 2001. Development of molecular methods for identification of Schizophyllum commune from clinical samples. J. Clin. Microbiol. 39: 23912396.

8. Chen, Y. Q., Wang, N., Qu, L. H., Li, T. H. and Zhang, W. M. 2001. Determination of the anamorph of Cordyceps sinensis inferred from the analysis of the ribosomal DNA internal transcribed spacers and ITS15.8S-ITS2 rDNA. Biochem. Syst. Ecol. 29: 597-607.

9. Chen, Y. Q., Wang, N., Zhou, H. and Qu, L. H. 2002. Differentiation of medicinal Cordyceps species by rDNA ITS sequence analysis. Planta. Med. 68: 635-639.

10. Eisenga, B. H., Trestrail III, J. H. and Emerson, T. S. 1998. Identification of unknown mushrooms: the good, the bad, and the ugly. J. Toxicol. Clin. Toxicol. 36: 635636.

11. Henry, T., Iwen, P. C. and Hinrichs, S. H. 2000. Identification of Aspergillus species using internal transcribed spacer regions 1 and 2. J. Clin. Microbiol. 38: 1510-1515.

12. Jackson, C. J. 2001. Molecular identification and strain typing of dermatophyte fungi. Nippon Ishinkin Gakkai Zasshi 42: 7-10.

13. James, T. Y., Moncalvo, J. M., Li, S. and Vilgalys, R. 2001. Polymorphism at the ribosomal DNA spacers and its relation to breeding structure of the widespread mushroom Schizophyllum commune. Genetics 157: 149161.

14. Kelly, J. M. and Cox, R. A. 1982. The nucleotide sequence at the 3'-end of Neurospora crassa 18S-rRNA and studies on the interaction with 5S-rRNA. Nucleic Acids Res. 10: 6733-6745.

15. Lee, S. B. and Taylor, J. W. 1990. Isolation of DNA from fungal mycelia and single spores. In "PCR Protocols: A Guide to Methods and Applications". 2nd ed. pp. 315-322. Academic Press. San Diego, U. S. A.

16. Lott, T. J., Burns,B. M., Zancope-Oliveira, R., Elie, C. M. and Reiss, E. 1998. Sequence analysis of the internal transcribed spacer 2 (ITS2) from yeast species within the genus Candida. Curr. Microbiol. 36: 63-69.

17. Lue, M. Y., Chang, C. C. and Liou, J. J. 2002. Identification of Cordyceps species. Rept. Taiwan Sugar Res. Inst. 175-176: 51-58. 
18. Turenne, C. Y., Sanche, S. E., Hoban, D. J., Karlowsky, J. A. and Kabani, A. M. 1999. Rapid identification of fungi by using the ITS2 genetic region and an automated fluorescent capillary electrophoresis system. J. Clin. Microbiol. 37: 1846-1851.

19. Varga, J., Toth, B., Rigo, K., Teren, J., Hoekstra, R. F. and Kozakiewicz, Z. 2000. Phylogenetic analysis of Aspergillus section circumdati based on sequences of the internal transcribed spacer regions and the ITS15.8S-ITS2 rRNA gene. Fungal Genet. Biol. 30: 71-80.

20. Velegraki, A., Kambouris, M., Kostourou, A., Chalevelakis, G. and Legakis, N. J. 1999. Rapid extraction of fungal DNA from clinical samples for PCR amplification. Med. Mycol. 37: 69-73.

21. Vilgalys, R. and Hester, M. 1990. Rapid genetic identification and mapping of enzymatically amplified ribosomal DNA from several Cryptococcus species. J. Bacteriol. 172: 4238-4246.

22. Zhang, D., Yang, Y., Castlebury, L. A. and Cerniglia, C. E. 1996. A method for the large scale isolation of high transformation efficiency fungal genomic DNA. FEMS. Microbiol. Lett. 145: 261-265.

23. Chen, Y., Zhang, Y. P., Yang, Y. and Yang, D. 1999. Genetic diversity and taxonomic implication of Cordyceps sinensis as revealed by RAPD markers. Biochem. Genet. 37: 210-213.
24. Chen, Y., Wang, W., Yang, Y. B., Su, Y. P., Zhang, L., Xiong, Z., He, C. S. and Yang, D. 1997. Genetic divergence of Cordyceps sinensis as estimated by random amplified polymorphic DNA analysis. Acta. Genet. Sinica. 24: 410-416.

25. Chomczynski, P., Mackey, K., Drews, R. and Wilfinger, W. 1997. DNAzol: a reagent for the rapid isolation of genomic DNA. Biotechniques 22: 550-553.

26. Chang, Y. H. 2002. Molecular systematics of Antrodia cinnamomea. Master Thesis, Graduate Institute of Agricultural Chemistry, National Taiwan University, Taiwan, R. O. C.

27. Johannesson, H., Renvall, P. and Stenlid, J. 2000. Taxonomy of Antrodiella inferred from morphological and molecular data. Mycol. Res. 104(1): 92-99.

28. Williams, D. W., Wilson, M. J., Lewis, M. A. O. and Potts, A. J. C. 1995. Identification of Candida species by PCR and restriction fragment length polymorphism analysis of intergenic spacer regions of ribosomal DNA. J. Clin. Microbiol. 33: 2476-2479. 